\title{
Effect of Argon Plasma on the Float Glass Surface
}

\author{
M. TuletA* \\ Institute of Physics, Technical University of Cracow, Podchorążych 1, 30-084 Cracow, Poland
}

Both surfaces of a commercial float glass were treated simultaneously by a low-temperature argon plasma generated by an inductively coupled rf power supply. The effect of plasma processing on the outer surface composition of both sides of the glass was analysed by means of the ion scattering spectroscopy technique. The observed recomposition of the outer surface atoms was interpreted as a result of the action of the thermal and electric fields created by the plasma on particular glass constituents.

PACS: 81.05.Kf, 52.77.-j, 82.80.-d

\section{Introduction}

Nowadays float glass is one of the most important kinds of commercial glass because it is widely used in modern architecture, automotive industry and optoelectronics for construction of liquid crystalline displays (LCDs) and plasma display panels (PDPs). Float glass is a flat, multicomponent oxide glass produced in the float process, in which a ribbon of glass is formed by floating of the glass melt on a bath of molten tin in a hot reducing atmosphere. Consequently, the bottom surface (called the tin side) and the top surface (called the atmosphere side) differ in the chemical composition and structure from those of the bulk. So, the float manufacturing process causes compositional changes of both surfaces. Also, a number of other processes, e.g. heat treatment, chemical reactions under ambient conditions, ion or electron irradiation and action of electric field modify the surface composition [1-8]. Because the state of the surface determines many physico-chemical properties of the glass, such as chemical durability, adhesion, mechanical strength, hardness, surface electric conductivity, refractive index and others [1, 9-13], it is important to investigate the above processes. Especially, plasma processing connecting the interaction of charge particles and thermal processing seems to be promising in modification of the glass surface.

In our earlier paper we observed a distinct dealkalization effect of the float glass surface caused by plasma treatment [14]. In the present study we describe the recomposition of the outer surface atoms induced by the argon plasma.

\section{Experimental}

In this experiment the cuboid samples $\left(5 \times 5 \times 4 \mathrm{~mm}^{3}\right)$ of commercial soda-lime-silica float glass with a basic

\footnotetext{
* e-mail: M.Tuleta@cyf-kr.edu.pl
}

composition of $\mathrm{SiO}_{2}$ 72.6, $\mathrm{Na}_{2} \mathrm{O}$ 13.0, $\mathrm{CaO}$ 8.4, $\mathrm{MgO}$ 4.0, $\mathrm{Al}_{2} \mathrm{O}_{3}$ 1.0, others 1.0 (in wt\%) were exposed to a low-temperature argon plasma.

The plasma was generated in an electrodeless reactor with an inductive discharge as described in the earlier paper [14].

Both sides of the glass were treated simultaneously with the argon plasma under the same plasma conditions. It was possible due to placing of two identical samples on an adjustable vertical quartz holder located on the axis of the reactor tube in such a manner that the atmosphere side of the first sample and the tin side of the second one were exposed to the plasma.

The atomic composition of the outer surface was determined by the low-energy ion scattering spectroscopy (ISS) technique. For this purpose, a cylindrical mirror analyser (CMA)-type ISS spectrometer (Kratos, Inc.) was used. The spectra for a constant scattering angle of $138^{\circ}$ were registered using a normally incident $1.5 \mathrm{keV}$ ${ }^{4} \mathrm{He}^{+}$beam. The ion beam was focused to an about $1 \mathrm{~mm}$ diameter spot with a current of $\approx 0.2 \mu \mathrm{A}$. The irradiation time needed to obtain the spectrum was about $30 \mathrm{~s}$. In order to reduce the build-up of positive surface charge the surface was flooded with electrons emitted from a heated tungsten filament placed near the sample surface.

\section{Results and discussion}

First, both surfaces of the float glass without plasma treatment were analysed by means of ion scattering spectroscopy. The aim of this analysis was to obtain information about the distribution of the outermost surface atoms, which was possible due to the monolayer sensitivity of ISS. It was interesting to see how the float manufacturing process and later storage of the glass influence its surface composition. Also, knowledge about the state of the surface was necessary to analyse properly surface changes caused by plasma treatment. 


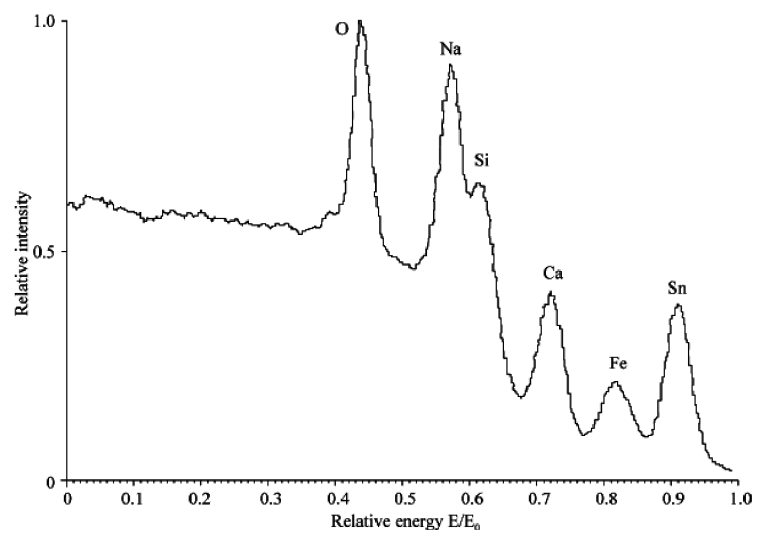

Fig. 1. ISS spectrum for the atmosphere side of untreated float glass.

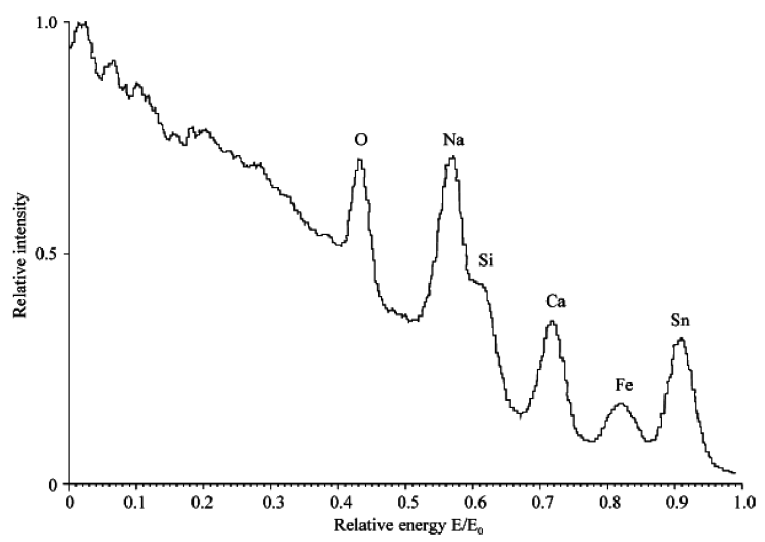

Fig. 2. ISS spectrum for the tin side of untreated float glass.

The ISS spectra of both surfaces are shown in Fig. 1 (atmosphere side) and Fig. 2 (tin side). The peaks are attributed to major components of the glass: oxygen, silicon, sodium and calcium. The lack of magnesium and aluminium peaks is connected probably with an overlap of these peaks with sodium and silicon ones, respectively. The spectra show also the presence of iron (small amount of this element is in the glass batch) and tin which is incorporated into the glass during its production. The peak positions do not correspond strictly with the energies predicted by the binary elastic collision model but are shifted towards higher energies. This fact can result from insufficient neutralisation of a positive charge deposited by the incident ion beam. Similar effect was described in Ref. [15].

The comparison of the spectrum characterising the tin side with the spectrum of the atmosphere side indicates that the first one has a larger low-energy tail. This can be related to the sodium enrichment on the tin side and its depletion on the atmosphere side as the secondary ion mass spectroscopy (SIMS) in-depth profiles show [14]. Sodium due to its low reionisation threshold $(\leq 200 \mathrm{eV})[16]$ can reionise the neutralised helium ions backscattered from deeper layers of the sample, which causes that they reach a detector with lower energy. Also, hydrocarbons presented on both surfaces can influence the tails. The occurrence of low-energy signals on the tin side may be explained by sputtering of sodium ions and presumably tin and iron ions that are on the tin side in larger amounts $[14,17]$. The enhanced presence of sodium on the tin side lowers the work function of the surface, which can cause the decrease of the scattered ion intensities due to the resonant neutralisation of helium ions. This is a complication for quantitative analysis because the ISS signals of atoms depend on their chemical environment [16].

Both sides of the glass were treated simultaneously by the argon plasma with a gas pressure of 0.5 Torr and a flow rate of $270 \mathrm{sccm}$ (the standard cc per minute) for $60 \mathrm{~min}$ at a temperature of $700^{\circ} \mathrm{C}$. After plasma treatment the samples were analysed by means of the ISS.

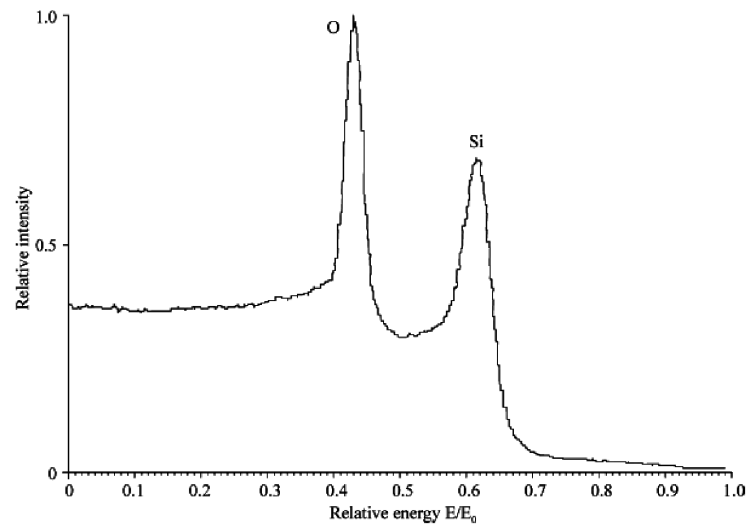

Fig. 3. ISS spectrum for the atmosphere side after plasma treatment.

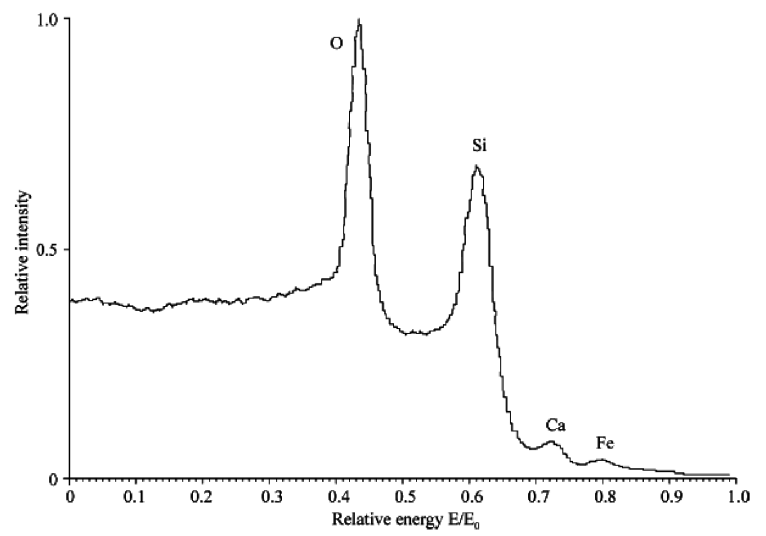

Fig. 4. ISS spectrum for the tin side after plasma treatment.

The spectra are presented in Fig. 3 (atmosphere side) and Fig. 4 (tin side). The spectrum of the atmosphere 
side shows the presence of oxygen and silicon and the opposite side indicates additionally the presence of calcium and iron. Such behaviour of glass constituents can be generally explained by complex interactions of plasma particles (electrons and argon ions) with glass ions. During plasma treatment the glass surface begins to charge negatively, because the electron flux reaching the surface is larger than the flux of the positive argon ions. Simultaneously, sodium ions and other glass modifiers having thermally increased mobility migrate towards the surface where they can be neutralised and leave the surface by evaporation. This effect causing sodium reduction in the glass surface was described in Ref. [14].

The occurrence of calcium and iron on the tin side can be connected with their lower mobility compared to sodium ions and the higher network connectivity of the near-surface region of the tin side, which could prevent the escape of these elements from the outer surface. Also, the oxidation state of iron alters its mobility $\left(\mathrm{Fe}^{3+}\right.$ is less mobile than $\mathrm{Fe}^{2+}$ ). Considering the factors influencing the spectra of both sides one should take into account the surface roughness and connected with this fact the shadowing and blocking effects. These effects and the neutralisation process cause that a quantitative compositional analysis of such type of the surface is not straightforward [16]. In the light of the results obtained for sodium [14] one can expect that the increase of the plasma temperature and the treatment time will cause also the reduction of other glass modifiers, which the presented ISS spectra show.

\section{Conclusions}

The commercial float glass was investigated by the ion scattering spectroscopy technique. The ISS spectra of untreated samples show the presence of main components of the glass, iron and tin. The low-energy tail, larger on the tin side, was observed. This effect was mainly caused by the sodium enrichment on the tin side.

Float glass shows the distinct surface recomposition under argon plasma processing due to thermal and electric fields created by the plasma. The ISS spectra of the atmosphere side indicate the presence of oxygen and silicon, which is characteristic of silica glass. The opposite side shows additionally calcium and iron due to the higher network connectivity on this side.

\section{Acknowledgments}

The author is grateful to Prof. C.G. Pantano for making it possible to carry out these measurements at The Pennsylvania State University and A.M. Then for experimental help.

\section{References}

[1] L. Holland, The Properties of Glass Surfaces, Chapman and Hall, London 1966.

[2] S. Takeda, R. Akiyama, H. Hosono, J. Non-Cryst. Solids 311, 273 (2002).

[3] S. Fearn, D.S. McPhail, R.J.H. Morris, M.G. Dowsett, Appl. Surf. Sci. 252, 7070 (2006).

[4] T. Lombardo, A. Chabas, R.-A. Lefevre, M. Verita, F. Geotti-Bianchini, Glass Technol. 46, 271 (2005).

[5] N.J. Smith, C.G. Pantano, J. Am. Ceram. Soc. 91 , 736 (2008).

[6] M.H. Modi, G.S. Lodha, M.K. Tiwari, S.K. Rai, C. Mukharjee, P. Magudapathy, K.G.M. Nair, R.V. Nandedkar, Nucl. Instrum. Methods Phys. Res. B 239, 383 (2005).

[7] G. Battaglin, E. Cattaruzza, F. Gonella, G. Mattei, P. Mazzoldi, C. Sada, X. Zhang, Nucl. Instrum. Methods Phys. Res. B 166-167, 857 (2000).

[8] G. Battaglin, G. Della Mea, G. De Marchi, P. Mazzoldi, A. Miotello, M. Guglielmi, J. Phys. Coll. C9 43, C9-645 (1982).

[9] A. Gorokhovsky, K. Matazov, J.I. Escalante-Garcia, J. Non-Cryst. Solids 291, 43 (2001).

[10] J.A. Howell, J.R. Hellmann, C.L. Muhlstein, J. Non-Cryst. Solids 354, 1891 (2008).

[11] D.I. Lee, Y.K. Lee, H.S. Lee, J. Mater. Sci. 27, 2908 (1992).

[12] P. Mazzoldi, J. Non-Cryst. Solids 120, 223 (1990).

[13] P.D. Townsend, N. Can, P.J. Chandler, B.W. Farmery, R. Lopez-Heredero, A. Peto, L. Salvin, D. Underdown, B. Yang, J. Non-Cryst. Solids 223, 73 (1998).

[14] M. Tuleta, Vacuum 54, 41 (1999).

[15] J.F. Kelso, C.G. Pantano, Surf. Interface Anal. 7, 228 (1985).

[16] H.H. Brongersma, M. Draxler, M. de Ridder, P. Bauer, Surf. Sci. Rep. 62, 63 (2007).

[17] Y. Hayashi, R. Akiyama, M. Kudo, Surf. Interface Anal. 31, 87 (2001). 